\title{
Research and Design of MEMS SINS/GPS Integrated Navigation System Based on Adaptive Filter
}

\author{
Gaowei Zhang ${ }^{1,2, a}$, Xiaoyu Zhang ${ }^{1}$, Chunlei Song ${ }^{1}$ and Tingting Wang ${ }^{2}$ \\ ${ }^{1}$ Sch. of Auto., Beijing Inst. of Technol., Beijing, China \\ ${ }^{2}$ Unit 91404 of the Chinese PLA, Qinhuangdao, China \\ aemail: zhanggaowei001@126.com
}

Keywords: MEMS; GPS; integrated navigation; adaptive filter.

\begin{abstract}
A MIMU/GPS integrated navigation system principle prototype is designed, and the structure of the system is introduced by different module. To handle the influence of Kalman filter parameters on system filtering performance (Including the system noise variance matrix $Q$ and measurement noise covariance matrix R), adaptive estimation Kalman filter is designed. The test results show that satisfactory performance can be obtained using adaptive estimation techniques for the low-cost MIMU/GPS integrated navigation.
\end{abstract}

\section{Introduction}

Strap-down inertial navigation system (SINS) is a fully autonomous navigation system, which is widely used in aircraft, military navigation, land mapping, mineral exploration and other fields. Over the last decade, with the development with the development of microelectronics and micro-mechanical technology, micro-electromechanical systems (MEMS) inertial devices quickly developed. MEMS gyroscopes and accelerometers constitutes a miniature inertial measurement unit (MIMU), The new MIMU has the feature of low cost, small size, low power consumption, high reliability and ability to adapt to the environment, it is one of the main development direction of the inertial technologies in the future. Currently, such MIMU's main problem is that MEMS sensor noise level is high, and most MEMS measurement accuracy is generally not satisfying. The algorithm of MIMU-based inertial navigation system (INS) uses the principle of dead reckoning, Integral operations rapid accumulate the navigation error, and thus unable to meet the requirements of high-precision navigation system indicators work long hours.

The Integrated Navigation is an effective way to solve this SINS navigation problem. GPS (Global Positioning System) provides global, round-the-clock high-precision navigation and positioning information. Its shortcoming is its vulnerability to interference; when the carrier doing maneuverable flight or obscured by terrain, GPS navigation information error is big or even be interrupted. It also has a low update rate which cannot meet the requirements of high maneuverability dynamic and real-time navigation. A combination of both can use GPS's long-term stability and high precision to make up MIMU's disadvantage of error accumulates with time, while MIMU's short-term autonomy can make up the GPS signal quality problems. Give full play to their respective advantages, and will greatly improve the accuracy and reliability of navigation and positioning.

\section{System hardware structure and design}

System architecture. The hardware side is mainly composed of the following components: The MIMU data acquisition modules, GPS data acquisition module, navigation solver module, power module and so on. Its structure is shown in Fig. 1.

MIMU acquisition module. The MIMU has three LCG50 gyroscopes for angular velocity acquisition, and a triaxial accelerometer model 2430. The whole MIMU is installed in a Thin-walled framework base, three gyros are orthogonally placed and all input axes parallel to accelerometer's 
sensitive axis. The MIMU has a reasonable layout, compact structure, small size, light weight, and can be used alone as the testing and use of the inertial navigation unit.

MIMU output signal through the low-pass filter, a signal conditioning amplifier circuit, and then the A / D conversion, the signal is converted to digital input to the MCU, and stored in the dual-port RAM for DSP navigation solution.

The analog filter using LINEAR's integrated analog filter chip LTC1068, The chip integrates four of the same second-order filter, and filtering band can be adjusted by changing the input clock frequency, it is able to realize second-order low-pass or high-pass, band-pass and band-stop function. This article uses three LTC1068 for gyro and accelerometer signals second-order low pass filtering, cutoff frequency is designed to be $100 \mathrm{~Hz}$, thus it effectively filter out the impact of the high-frequency noise meanwhile meet the system dynamic performance.

ADC data acquisition using ADI's Analog-to-Digital Converter AD7606-6 is 16 bit, 6 channels, 200 kSPS simultaneous sampling Analog-to-Digital Data Acquisition system (DAS). The parts contains analog input clamp protection, 2nd order anti-alias filter, track and hold amplifier, 16-bit charge redistribution successive approximation $\mathrm{ADC}$, flexible digital filter, $2.5 \mathrm{~V}$ reference and reference buffer and high speed serial and parallel interfaces. This ADC operate from a single $5 \mathrm{~V}$ supply, its anti-alias filter has a $3 \mathrm{~dB}$ cut off frequency of $22 \mathrm{kHz}$ and provides $40 \mathrm{~dB}$ anti-alias rejection when sampling at 200ksps. The flexible digital filter is pin driven, yields improvements in SNR, and reduces the $3 \mathrm{~dB}$ bandwidth.

GPS acquisition module. GPS receiver is SUPERSTAR II. The SUPERSTAR II features 12-channel code and carrier phase tracking for increased accuracy. Position, velocity, time (PVT) and raw carrier phase measurements output are available at rates of up to $5 \mathrm{~Hz}$. It is also characterized by small package and low power consumption. GPS's control and data output via RS232 serial port, use MCU LM3S1811 to execute its data acquisition and control. On the one hand receive GPS output packet data, and identify, check and pre-processing of the message, after that store the results in dual-port RAM; on the other hand MCU sends control packets to the OEM board to control the GPS receiver to output the desired messages.

After the GPS receiver complete its self-test, according to the requirements of the navigation system, MCU first read the data in the dual-port RAM, send control messages to the GPS, initialize the OEM board, allowing the GPS receiver to output the desired message. The subsequent GPS receiver automatically received GPS RF signal from its antenna, after conversion, amplification, correlation, the mixer with TTL transform a series of processing, measure out the propagation time of signal travel from the satellite to the between the antennas, interprets the navigation message issued by the GPS satellite, and finally output data through the serial port.

DSP navigation solver module. The DSP navigation solver module uses a floating-point DSP TMS320C6713B as the core CPU, operating at $300 \mathrm{MHz}$, the C6713B delivers up to $1800 \mathrm{MFLOPS}$, 2400 MIPS, and 600 MMACS. The C6713B has a rich peripheral set that includes two McBSPs, two I2C buses, GPIO module, two general-purpose timers, a HPI, and a glueless EMIF capable of interfacing to SDRAM, SBSRAM, and asynchronous peripherals. Its main task is complete inertial navigation and integrated navigation solution, Kalman filtering and all other data processing tasks.

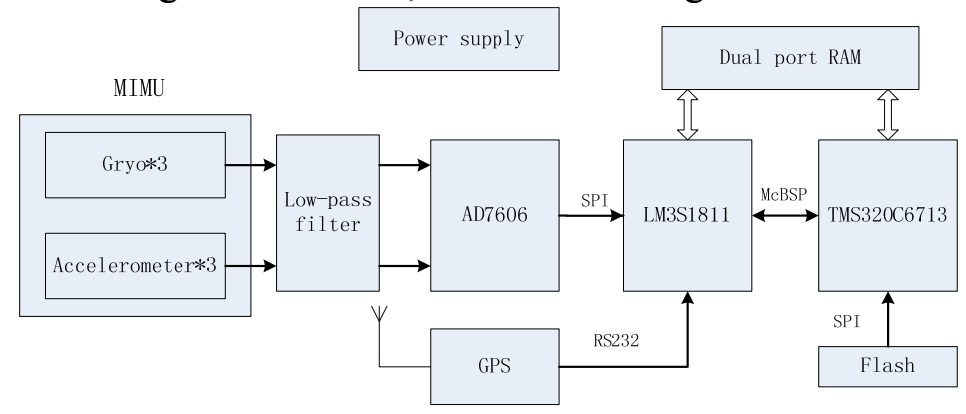

Fig. 1. System architecture

\section{Design and realization of software}


MIMU / GPS system filter model and basic filtering equation. The system chose "EAST -NORTH-UP" coordinate system as the navigation coordinate system. Random drift error of the gyroscopes and accelerometers random bias errors are modeled as white noise with a combination of a first-order Markov process, then the equation of state of MIMU / GPS integrated navigation system is:

$$
\begin{aligned}
& \dot{X}(t)=F(t) X(t)+G(t) W(t) \\
& X(t)=\left[\begin{array}{lllllllllllllll}
\phi_{E} & \phi_{N} & \phi_{U} & \delta_{V E} & \delta_{V N} & \delta_{V U} & \delta_{\lambda} & \delta_{\varphi} & \delta_{h} & \varepsilon_{x} & \varepsilon_{y} & \varepsilon_{z} & f_{x} & f_{y} & f_{z}
\end{array}\right]^{\mathrm{T}}
\end{aligned}
$$

Where, $\begin{array}{llllllll}\phi_{E} & \phi_{N} & \phi_{U} & \text { is } 3 \text { platform misalignment angle, } \delta_{V E} & \delta_{V N} & \delta_{V U} & \text { is three-dimensional }\end{array}$ velocity error, $\varepsilon_{x} \quad \varepsilon_{y} \quad \varepsilon_{z}$ is first-order Markov drift of 3-axis gyroscope, $f_{x} f_{y} f_{z}$ is first-order Markov bias of 3-axis accelerometer, $F(t)$ is system transfer matrix, $W(t)$ is system noise.

$$
\begin{aligned}
& W(t)=\left[\begin{array}{llllll}
\omega_{\epsilon \mathrm{x}} & \omega_{\epsilon \mathrm{y}} & \omega_{\epsilon z} & \omega_{\mathrm{fx}} & \omega_{\mathrm{fy}} & \omega_{\mathrm{fz}}
\end{array}\right]^{\mathrm{T}} \\
& G(\mathrm{t})=\left[\begin{array}{cc}
\mathrm{C}_{\mathrm{b}}^{\mathrm{n}} & 0_{3 \times 3} \\
0_{3 \times 3} & \mathrm{C}_{\mathrm{b}}^{\mathrm{n}} \\
0_{9 \times 3} & 0_{9 \times 3}
\end{array}\right], \mathrm{F}(\mathrm{t})=\left[\begin{array}{cc}
\mathrm{F}_{\mathrm{N}} & \mathrm{F}_{\mathrm{S}} \\
0_{6 \times 6} & \mathrm{~F}_{\mathrm{M}}
\end{array}\right], \mathrm{F}_{\mathrm{S}}=\left[\begin{array}{cc}
\mathrm{C}_{\mathrm{b}}^{\mathrm{n}} & 0_{3 \times 3} \\
0_{3 \times 3} & \mathrm{C}_{\mathrm{b}}^{\mathrm{n}} \\
0_{3 \times 3} & 0_{3 \times 3}
\end{array}\right], \mathrm{F}_{\mathrm{M}}=\left[0_{6 \times 15}\right]
\end{aligned}
$$

Where, $F_{N}$ is corresponding to the nine basic navigation parameters array.

System measurement equation is:

$$
Z(t)=H(t)+V(t)
$$

$Z(t)$ is system measurement vector, $H(t)$ is measurement matrix, $V(t)$ is measurement noise vector.

$$
\begin{aligned}
& \mathrm{V}(\mathrm{t})=\left[\begin{array}{llllll}
\mathrm{V}_{\delta \mathrm{VE}} & \mathrm{V}_{\delta \mathrm{VN}} & \mathrm{V}_{\delta \mathrm{VU}} & \mathrm{V}_{\delta \lambda} & \mathrm{V}_{\delta \varphi} & \mathrm{V}_{\delta \mathrm{h}}
\end{array}\right]^{\mathrm{T}} \\
& H(t)=\left[\begin{array}{lllll}
0_{6 \times 3} & \operatorname{diag}\left(1,1,1, R_{m}, R_{n}, \cos L, 1\right) & 0_{6 \times 3}
\end{array}\right]
\end{aligned}
$$

Discrete state equation and measurement equation, we got:

$$
X_{k}=\Phi_{k, k-1} X_{k-1}+\Gamma_{k, k-1} W_{k-1}, Z_{k}=H_{k} X_{k}+V_{k}
$$

For equations (4), Kalman filter equations are:

$$
\begin{aligned}
& \hat{X}_{k, k-1}=\Phi_{k, k-1} \hat{X}_{k-1}, \hat{X}_{k}=X_{k, k-1}+K_{k}\left[Z_{k}-H_{k} \hat{X}_{k, k-1}\right], K_{k}=P_{k, k-1} H_{k}^{T}\left[H_{k} P_{k, k-1} H_{k}^{T}+R_{k}\right]^{-1}, \\
& P_{k, k-1}=\Phi_{k, k-1} P_{k-1} \Phi_{k, k-1}^{T}+Q_{k-1}, P_{k}=\left[I-K_{k} H_{k}\right] P_{k, k-1}
\end{aligned}
$$

The influence of system noise covariance matrix $Q$ and measurement noise covariance matrix R on MIMU / GPS system filtering performance. In the normal Kalman filter design, it is assumed that the observation noise and system noise model is accurate, however, in practical application is difficult to model system noise and measurement noise precisely. From equation (5) it can roughly be seen that at time $K$, the gain matrix $K_{k}$ is proportional to the real system noise variance $Q_{K}$, and is inversely proportional to the real measured variance $R_{k}$, so $Q_{K}$ and $R_{k}$ have a significant impact on the filtering performance. In MIMU / GPS integrated navigation Kalman filter, wrong $Q_{K}$ may lead to filter divergence. For $R_{k}$, when it is too high, the filter may be diverging; when it is too low, it may cause large errors.

Adaptive filter design. In order to robustly deal with the uncertainty of the measurement noise and system noise, the adaptive filter is used to identify the $Q_{K}$ and $R_{k}$. Largest natural estimation method is adaptive estimation within a Kalman filter framework. Its basic idea is the use of the residuals of the measured values and status values to correct the measurement noise and system noise.

$Q_{K}$ Adaptive Design. In an ordinary Kalman filter, it is assumed that the system noise $\omega_{k}$ is zero mean Gaussian white noise, i.e. $\omega_{k}$ obey the normal distribution of zero mean and variance $Q$ (i.e. $\mathrm{N}(0$, $\left.Q_{K}\right)$ ). Adaptive estimation uses the prediction of the state value and the update value of status to update the value of $Q_{K}$. The update equation of $Q_{K}$ is: 


$$
Q^{*}=\Delta X_{k} \Delta X_{k}^{T}+P_{k}^{-}-P_{k}^{+}-Q_{k}^{-}, \Delta X_{k}=X_{k}^{-}-X_{k}^{+}
$$

Where, $Q^{*}$ is update value of the system noise covariance matrix, $\Delta X_{k}$ is state estimation residuals, $X_{k}^{+}$is state estimate, $X_{k}^{-}$is status predictive value, $P_{k}^{+}$is State covariance matrix estimate, $P_{k}^{-}$is State covariance matrix predictive value, $Q_{k}^{-}$is the noise covariance matrix of current expectations system.

Equation (6) is the heart of adaptive filter, if the state estimation residuals $\Delta X_{k}$ is large, Then the filter is not a good predictor of the future state, when the filter is convergent, state estimation residuals will reduce. Equation (6) can also be written as:

$$
Q^{*}=\Delta X_{K} \Delta X_{K}^{T}-\left[P_{K}^{-}-\left(P_{K}^{+}+Q_{K}^{-}\right)\right]
$$

Which is equivalent to:

$$
Q^{*}=\Delta X_{K} \Delta X_{K}^{T}-\left[P_{K}^{-}-\left(\Phi_{\mathrm{k}, \mathrm{k}-1} P_{K-1}^{+} \Phi_{\mathrm{k}, \mathrm{k}-1}^{\mathrm{T}}\right)\right]
$$

Through Low-pass filter Update value $Q^{*}$ and current value of the system noise variance $Q_{K}$ are combined to yield:

$$
Q_{K}^{+}=Q_{K}^{-}+\frac{1}{L_{Q}}\left(Q^{*}-Q_{K}^{-}\right)
$$

Where, $L_{Q}$ is the window size, the performance of the adaptive filter is sensitive to the choice of $L_{Q}$, should be selected according to the actual situation in the practical application.

$R_{k}$ Adaptive Design. Measurement noise identification is similar to system noise identification, define the measurement noise vector $\mathrm{v}$ obey a zero-mean Gaussian white noise, so $\mathrm{v}$ obey normal distribution with zero mean and variance $R$ (i.e. $\mathrm{N}\left(0, R_{k}\right)$ ), measurement noise covariance matrix update equation is:

$$
R^{*}=\Delta Y_{K} \Delta Y_{K}^{T}-H_{K} P_{k}^{+} H_{K}^{T}, \Delta Y_{k}=Y_{K}-Y_{k}^{+}
$$

Where, $\Delta Y_{k}$ is measurement residuals. Similar to $Q$ Adaptive Estimation, Through Low-pass filter Update value $R^{*}$ and current value of the system noise variance $R_{k}$ are combined to yield:

$$
R_{K}^{+}=R_{K}^{-}+\frac{1}{L_{R}}\left(R^{*}-R_{K}^{-}\right)
$$

Similarly, the performance of the adaptive filter is sensitive to the choice of $L_{R}$, should be selected according to the actual situation in the practical application.

\section{Analysis and comparison of the test performance}

Use the designed MIMU/GPS navigation system verified the adaptive Kalman filter algorithm, the accuracy under static conditions was tested. According to the result, compared with ordinary filter, adaptive Kalman filter algorithm has better robustness. The test result of the comparison is shown in Fig. 2 to Fig. 7 below.

As can be seen from the Figures, MIMU/GPS integrated navigation system has better performance using adaptive Kalman filter. Position accuracy is better than $4.5 \mathrm{~m}$, speed accuracy is better than $0.08 \mathrm{~m} / \mathrm{s}$, pitch angle and roll angle accuracy is better than $0.18^{\circ}$, yaw angle accuracy is better than $0.45^{\circ}$.

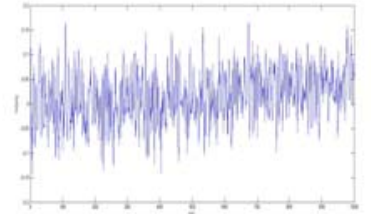

Fig. 2. Adaptive estimation integrated navigation velocity error along $\mathrm{x}, \mathrm{y}$ axes
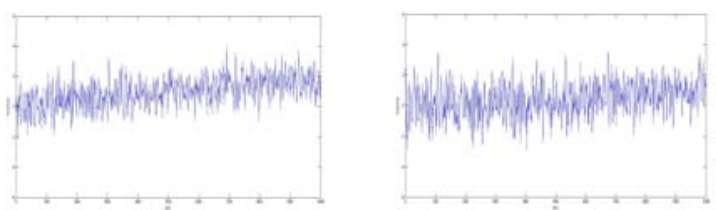

Fig. 3. Ordinary integrated navigation velocity error along $\mathrm{x}, \mathrm{y}$ axes 


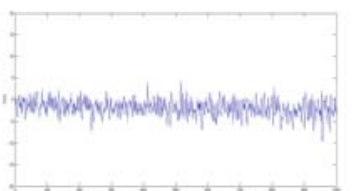

Fig. 4. Adaptive estimation integrated navigation along $\mathrm{x}, \mathrm{y}$ axes position error
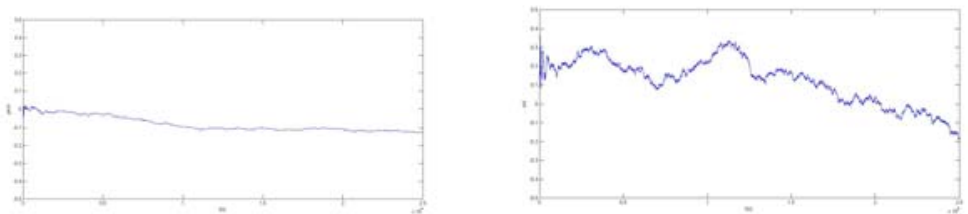

Fig.6 Adaptive estimation integrated navigation attitude error (pitch, roll and yaw respectively)
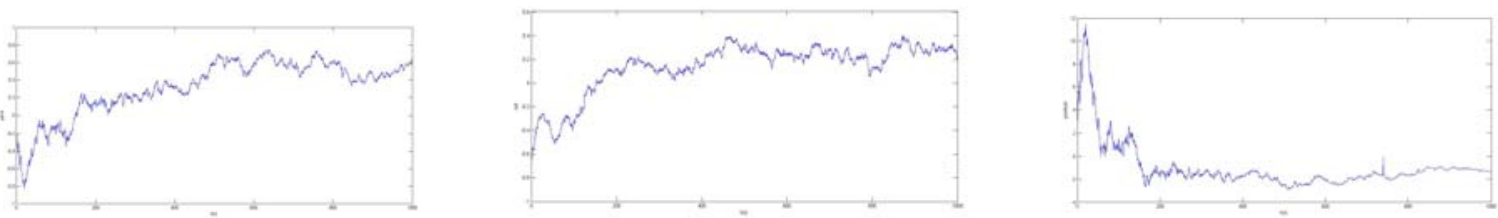

Fig.7 Ordinary integrated navigation attitude error(pitch, roll and yaw respectively)

\section{Conclusions}

Designed and realized MIMU/GPS integrated navigation system prototype, To deal with the problem of system performance is impacted by system noise variance matrix $Q$ and measurement noise covariance matrix $R$, adaptive estimation technique is adopted to estimate the Kalman filter parameters. Experiments verified that adaptive Kalman filter MIMU/GPS integrated navigation system has better performance.

\section{References}

[1]M.M Zhao, J.H Zhu and X.H Feng: The Study of MIMU/GPS Integrated Navigation System Based on UKF (Control \& Automation 2009 In Chinese).

[2]X.J Yang, G.N Yuan and L.C Xu: The Research of MIMU System Design (Journal of Projectiles,Rockets,Missiles and Guidance 2006).

[3]Y.F Ma: Design of MIMU/ GPS Navigation Computer (Computer Measurement \& Control 2009).

[4]C.P Shang, X.J Zhang and J Wang: The Application Study of Integrated Navigation System Based MIMU/GPS (Control \& Automation 2010).

[5]H.J Zhang, B.Y Liao and H.Yuan: MIMU/GPS Integrated Navigation System Based on Strong Tracking Filter (Computer Simulation 2011).

[6]T.J.Cai, Y.Liu, J.Song and Y.H Zhu: Embedded integrated GPS/MIMU/compass navigation system (Chinese Journal of Scientific Instrument 2010).

[7]X.M.Zhou, L.Su; L.D.Guo, Y.Liang, "Integrated Navigation System Study Based on Multiscale Recursive Fusion Estimation” ISA,2009, pp.1-4.

[8]Q.P.Wu, Z.P.Gao, Y.L.Wang, "Study on GPS/DR/MM integrated navigation system for vehicle based on DSP", IEEE 2002 International Conference, 2002 , Page(s): 1591 - 1595 vol.2

[9]D.X.Xu, L.Wang, G.C.Li, T.Ma, "Study of strong tracking augmented unscented kalman filter in integrated navigation system". Intelligent Control and Automation (WCICA), 2012 , Page(s): 5112 5115. 